\title{
PCM1 Gene
}

National Cancer Institute

\section{Source}

National Cancer Institute. PCM1 Gene. NCI Thesaurus. Code C97900.

This gene is involved in centrosome formation. 